\title{
Variations of Infinite Derivative Modified Gravity
}

Ivan Dimitrijevic, Branko Dragovich, Zoran Rakic and Jelena Stankovic

\begin{abstract}
We consider nonlocal modified Einstein gravity without matter, where nonlocal term has the form $P(R) \mathcal{F}(\square) Q(R)$. For this model, in this paper we give the derivation of the equations of motion in detail. This is not an easy task and presented derivation should be useful to a researcher who wants to investigate nonlocal gravity. Also, we present the second variation of the related Einstein-Hilbert modified action and basics of gravity perturbations.
\end{abstract}

\section{Introduction}

General relativity 35, which is Einstein theory of gravity, is dominant theory of gravitational phenomena for more than last hundred years. It is one of the most attractive and phenomenologically successful physical theories. General relativity is perfectly confirmed in the Solar system. Among many

Ivan Dimitrijevic

Faculty of Mathematics, University of Belgrade, Belgrade, Serbia, e-mail: ivandematf.bg.ac.rs

Branko Dragovich

Institute of Physics, University of Belgrade, Belgrade, Serbia,

Mathematical Institute, Serbian Academy of Sciences and Arts, Belgrade, Serbia email: dragovich@ipb.ac.rs

Zoran Rakic

Faculty of Mathematics, University of Belgrade, Belgrade, Serbia, e-mail: zrakicematf.bg.ac.rs

Jelena Stankovic

Teacher Education Faculty, University of Belgrade, Belgrade, Serbia e-mail: jelenagg@gmail.com 
important predictions are gravitational red shift, gravitational lensing, gravitational waves and black holes.

Although very successful, Einstein gravity is not a complete and final theory of gravitational phenomena. According to its cosmological solutions, the universe contains initial singularity. This singularity is a serious and still unsolved problem, which requires an adequate Einstein gravity modification. General relativity predicts that the universe contains about $68 \%$ of dark energy, $27 \%$ of dark matter and only about $5 \%$ of visible matter. However, dark energy and dark matter are not yet experimentally confirmed. Also, Einstein gravity has not been verified at very large cosmic scales. Hence, some cosmological predictions, including energy/matter content of the universe, based on Einstein gravity should be taken with caution. One has also to mention problem of quantization of general relativity. Hence, it follows that Einstein gravity has some theoretical and phenomenological problems in ultraviolet and infrared regions.

Unfortunately, a new theoretical principle which would tell us which is right direction to modify gravity is not yet uncovered. Hence, there are many modifications of Einstein gravity, which are motivated by problems in quantum gravity, string theory, astrophysics and cosmology (for a review, see [9, 30, 32, 33, 31]).

One of recent and very promising directions of research is nonlocal modified gravity with its applications to cosmology (as a review, see [30, 31, 36, 2, 15, 20]). Potentially there is a huge number of possibilities to construct a nonlocal gravity model by replacement of the scalar curvature $R$ in the EinsteinHilbert action by a scalar function $F\left(R, \square, \square^{-1}, R_{\mu \nu} R^{\mu \nu}, R_{\mu \nu \alpha \beta} R^{\mu \nu \alpha \beta}, \ldots\right)$, where $\square=\nabla_{\mu} \nabla^{\mu}$ is d'Alembert operator and $\nabla_{\mu}$ denotes the covariant derivative. In this paper, nonlocality means that gravitational Lagrangian contains an infinite number of space-time derivatives, i.e. derivatives up to an infinite order in the form of d'Alembert operator $\square$, which is argument of an analytic function. Note that higher derivative gravity theories improve problems with quantization of general relativity, see, e.g. [34, 23, 27, 28].

In the sequel we consider nonlocal modification of gravity where EinsteinHilbert action contains an additional nonlocal term of the form $P(R) \mathcal{F}(\eta \square) Q(R)$, where $\eta$ is a parameter and $\eta \square$ is dimensionless. In fact we consider a class of nonlocal gravity models without matter given by the action

$$
S=\frac{1}{16 \pi G} \int_{M}(R-2 \Lambda+P(R) \mathcal{F}(\eta \square) Q(R)) \sqrt{-g} \mathrm{~d}^{4} x,
$$

where $M$ is a pseudo-Riemannian manifold of signature $(1,3)$ with metric $\left(g_{\mu \nu}\right), \mathcal{F}(\eta \square)=\sum_{n=0}^{\infty} f_{n}(\eta \square)^{n}, P$ and $Q$ are differentiable functions of the scalar curvature $R$ and $\Lambda$ is cosmological constant. Inspiration for an analytic form of the function $\mathcal{F}(\eta \square)$ comes from string theory, in particular from $p$ adic string theory, which is a part of $p$-adic mathematical physics, for a recent 
review see 21. The corresponding Einstein equations of motion have complex structure. In this paper we will present their derivation, because it is not an easy task and it should be useful to a reader interested in this subject. It is also useful to see [6. In order to obtain equations of motion for $g_{\mu \nu}$ we have to find the variation of the action (11) with respect to metric $g^{\mu \nu}$. In addition we also find the second variation of the action (11) and consider some cosmological perturbations. For simplicity, in the sequel we shall take $\eta=1$.

Before to proceed with derivation of equations of motion for the above model (1), it is worth to mention some other nonlocal models with inverse d'Alembert operator, i. e. with $\square^{-n}$, which are proposed to explain the late time cosmic acceleration without dark energy. Such models have the form

$$
S=\frac{1}{16 \pi G} \int \sqrt{-g}\left(R+L_{N L}\right) d^{4} x,
$$

where two typical examples are: $L_{N L}=R f\left(\square^{-1} R\right)$ (see a review 30, 36] and references therein), and $L_{N L}=-\frac{1}{6} m^{2} R \square^{-2} R$ (see a review [2] and references therein).

Nonlocal models with $\mathcal{F}(\square)=\sum_{n=0}^{\infty} f_{n} \square^{n}$ are mainly considered to improve general relativity in its ultraviolet region, unlike models with $\square^{-1}$ and $\square^{-2}$ which intend to modify gravity in its infrared sector. It may happen that there will be more than one modification of general relativity, which are valid at the different scales. Namely, any physical theory has a domain of validity, which depends on some conditions, including spatial scale and complexity of the system. It is natural that validity of general relativity is also restricted. At very short and very large cosmic distances may act different gravity theories.

Section 2 contains variation of curvature tensors from pseudo-Riemannian geometry, what is necessary for derivation of equations of motion for $g_{\mu \nu}$ in Sect. 3. Second variation of gravity modified action (11) is presented in Sect. 4. Basics of cosmic perturbations are subject of Sect. 5. Sect. 6 contains some concluding remarks.

\section{Variation of curvature tensors}

Let us start with a technical lemma:

Lemma 1. The following relations hold

$$
\begin{aligned}
\delta g & =g g^{\mu \nu} \delta g_{\mu \nu}=-g g_{\mu \nu} \delta g^{\mu \nu}, \\
\delta \sqrt{-g} & =-\frac{1}{2} g_{\mu \nu} \sqrt{-g} \delta g^{\mu \nu} \\
\delta \Gamma_{\mu \nu}^{\lambda} & =-\frac{1}{2}\left(g_{\nu \alpha} \nabla_{\mu} \delta g^{\lambda \alpha}+g_{\mu \alpha} \nabla_{\nu} \delta g^{\lambda \alpha}-g_{\mu \alpha} g_{\nu \beta} \nabla^{\lambda} \delta g^{\alpha \beta}\right),
\end{aligned}
$$


where $g$ is the determinant of the metric tensor.

Proof. Determinant $g$ can be written as

$$
g_{\mu \nu} G^{(\alpha, \nu)}=g \delta_{\mu}^{\alpha}
$$

where $G^{(\mu, \nu)}$ is the algebraic cofactor of the element $g_{\mu \nu}$.

Thus,

$$
g^{\mu \nu}=\frac{G^{(\mu, \nu)}}{g} .
$$

Since $G^{\mu, \nu}$ is independent of $g_{\mu \nu}$ we obtain the first part of the equation (3)

$$
\delta g=g g^{\mu \nu} \delta g_{\mu \nu} .
$$

Moreover from $g_{\mu \nu} g^{\mu \nu}=4$ and Leibniz rule we obtain $g^{\mu \nu} \delta g_{\mu \nu}=-g_{\mu \nu} \delta g^{\mu \nu}$, which completes the proof of (3).

To prove the equation (4) we proceed in the following way

$$
\delta \sqrt{-g}=-\frac{1}{2 \sqrt{-g}} \delta g=-\frac{1}{2} g_{\mu \nu} \sqrt{-g} \delta g^{\mu \nu} .
$$

The third equation is proved by

$$
\begin{aligned}
\nabla_{\lambda} g_{\mu \nu} & =\partial_{\lambda} g_{\mu \nu}-\Gamma_{\lambda \mu}^{\kappa} g_{\kappa \nu}-\Gamma_{\lambda \nu}^{\kappa} g_{\mu \kappa} \\
\delta \Gamma_{\mu \nu}^{\lambda} & =\frac{1}{2} \delta g^{\lambda \kappa}\left(\partial_{\mu} g_{\kappa \nu}+\partial_{\nu} g_{\mu \kappa}-\partial_{\kappa} g_{\mu \nu}\right)+\frac{1}{2} g^{\lambda \kappa}\left(\partial_{\mu} \delta g_{\kappa \nu}+\partial_{\nu} \delta g_{\mu \kappa}-\partial_{\kappa} \delta g_{\mu \nu}\right) \\
\delta \Gamma_{\mu \nu}^{\lambda} & =\frac{1}{2} \delta g^{\lambda \kappa}\left(\partial_{\mu} g_{\kappa \nu}+\partial_{\nu} g_{\mu \kappa}-\partial_{\kappa} g_{\mu \nu}\right)+\frac{1}{2} g^{\lambda \kappa}\left(\partial_{\mu} \delta g_{\kappa \nu}+\partial_{\nu} \delta g_{\mu \kappa}-\partial_{\kappa} \delta g_{\mu \nu}\right) \\
& =\frac{1}{2} \delta g^{\lambda \kappa}\left(\partial_{\mu} g_{\kappa \nu}+\partial_{\nu} g_{\mu \kappa}-\partial_{\kappa} g_{\mu \nu}\right)+\frac{1}{2} g^{\lambda \kappa}\left(\nabla_{\mu} \delta g_{\kappa \nu}+\nabla_{\nu} \delta g_{\mu \kappa}-\nabla_{\kappa} \delta g_{\mu \nu}\right) \\
& +g^{\lambda \kappa} \Gamma_{\mu \nu}^{\alpha} \delta g_{\kappa \alpha} \\
& =\frac{1}{2} g^{\lambda \kappa}\left(\nabla_{\mu} \delta g_{\kappa \nu}+\nabla_{\nu} \delta g_{\mu \kappa}-\nabla_{\kappa} \delta g_{\mu \nu}\right)
\end{aligned}
$$

In the last step we used $\delta g_{\mu \nu}=-g_{\mu \alpha} g_{\nu \beta} \delta g^{\alpha \beta}$. Using the same equation in every term of the last equation we obtain (5):

$$
\begin{aligned}
\delta \Gamma_{\mu \nu}^{\lambda} & =\frac{1}{2} g^{\lambda \kappa}\left(\nabla_{\mu}\left(-g_{\kappa \alpha} g_{\beta \nu} \delta g^{\alpha \beta}\right)+\nabla_{\nu}\left(-g_{\mu \alpha} g_{\kappa \beta} \delta g^{\alpha \beta}\right)-\nabla_{\kappa}\left(-g_{\mu \alpha} g_{\nu \beta} \delta g^{\alpha \beta}\right)\right) \\
& =-\frac{1}{2}\left(\delta_{\alpha}^{\lambda} g_{\beta \nu} \nabla_{\mu} \delta g^{\alpha \beta}+\delta_{\beta}^{\lambda} g_{\mu \alpha} \nabla_{\nu} \delta g^{\alpha \beta}-g_{\mu \alpha} g_{\nu \beta} \nabla^{\lambda} \delta g^{\alpha \beta}\right) \\
& =-\frac{1}{2}\left(g_{\nu \alpha} \nabla_{\mu} \delta g^{\lambda \alpha}+g_{\mu \alpha} \nabla_{\nu} \delta g^{\lambda \alpha}-g_{\mu \alpha} g_{\nu \beta} \nabla^{\lambda} \delta g^{\alpha \beta}\right) .
\end{aligned}
$$


Note, that $\delta \Gamma_{\mu \lambda}^{\lambda}=-\frac{1}{2} g_{\lambda \alpha} \nabla_{\mu} \delta g^{\lambda \alpha}$.

Lemma 2. The variation of Riemman tensor, Ricci tensor and scalar curvature satisfy the following relations

$$
\begin{aligned}
\delta R_{\mu \beta \nu}^{\alpha} & =\nabla_{\beta} \delta \Gamma_{\mu \nu}^{\alpha}-\nabla_{\nu} \delta \Gamma_{\mu \beta}^{\alpha}, \\
\delta R_{\mu \nu} & =\nabla_{\lambda} \delta \Gamma_{\mu \nu}^{\lambda}-\nabla_{\nu} \delta \Gamma_{\mu \lambda}^{\lambda}, \\
\delta R & =R_{\mu \nu} \delta g^{\mu \nu}-K_{\mu \nu} \delta g^{\mu \nu}, \\
\delta \nabla_{\mu} \nabla_{\nu} \psi & =\nabla_{\mu} \nabla_{\nu} \delta \psi-\nabla_{\lambda} \psi \delta \Gamma_{\mu \nu}^{\lambda},
\end{aligned}
$$

where $K_{\mu \nu}=\nabla_{\mu} \nabla_{\nu}-g_{\mu \nu} \square$.

Proof. The variation of Riemann tensor is obtained as follows

$$
\begin{aligned}
\delta R_{\mu \beta \nu}^{\alpha} & =\delta\left(\partial_{\beta} \Gamma_{\mu \nu}^{\alpha}-\partial_{\nu} \Gamma_{\mu \beta}^{\alpha}+\Gamma_{\mu \nu}^{\lambda} \Gamma_{\beta \lambda}^{\alpha}-\Gamma_{\mu \beta}^{\lambda} \Gamma_{\nu \lambda}^{\alpha}\right) \\
& =\partial_{\beta} \delta \Gamma_{\mu \nu}^{\alpha}-\partial_{\nu} \delta \Gamma_{\mu \beta}^{\alpha}+\delta \Gamma_{\mu \nu}^{\lambda} \Gamma_{\beta \lambda}^{\alpha}+\Gamma_{\mu \nu}^{\lambda} \delta \Gamma_{\beta \lambda}^{\alpha}-\Gamma_{\mu \beta}^{\lambda} \delta \Gamma_{\nu \lambda}^{\alpha} \\
& -\delta \Gamma_{\mu \beta}^{\lambda} \Gamma_{\nu \lambda}^{\alpha}-\Gamma_{\beta \nu}^{\lambda} \delta \Gamma_{\mu \lambda}^{\alpha}+\Gamma_{\beta \nu}^{\lambda} \delta \Gamma_{\mu \lambda}^{\alpha} .
\end{aligned}
$$

In the last equation first, third and fifth term combined give $\nabla_{\beta} \delta \Gamma_{\mu \nu}^{\alpha}$, and second, fourth and sixth term give $-\nabla_{\nu} \delta \Gamma_{\mu \beta}^{\alpha}$, so we proved (18). Equation (19) is obtained from the previous by contracting indices $\alpha$ and $\beta$.

To prove the equation (20) we begin with $R=g^{\mu \nu} R_{\mu \nu}$. Applying the operator $\delta$ to both sides yields

$$
\begin{aligned}
\delta R & =R_{\mu \nu} \delta g^{\mu \nu}+g^{\mu \nu} \delta R_{\mu \nu} \\
& =R_{\mu \nu} \delta g^{\mu \nu}+g^{\mu \nu}\left(\nabla_{\lambda} \delta \Gamma_{\mu \nu}^{\lambda}-\nabla_{\nu} \delta \Gamma_{\mu \lambda}^{\lambda}\right) \\
& =R_{\mu \nu} \delta g^{\mu \nu}-\frac{1}{2} g^{\mu \nu}\left(2 g_{\nu \alpha} \nabla_{\lambda} \nabla_{\mu} \delta g^{\lambda \alpha}-g_{\mu \alpha} g_{\nu \beta} \square \delta g^{\alpha \beta}-g_{\lambda \alpha} \nabla_{\nu} \nabla_{\mu} \delta g^{\lambda \alpha}\right) \\
& =R_{\mu \nu} \delta g^{\mu \nu}-\frac{1}{2}\left(2 \delta_{\alpha}^{\mu} \nabla_{\lambda} \nabla_{\mu} \delta g^{\lambda \alpha}-\delta_{\alpha}^{\nu} g_{\nu \beta} \square \delta g^{\alpha \beta}-g_{\lambda \alpha} \square \delta g^{\lambda \alpha}\right) \\
& =R_{\mu \nu} \delta g^{\mu \nu}-\frac{1}{2}\left(2 \nabla_{\mu} \nabla_{\nu} \delta g^{\mu \nu}-2 g_{\mu \nu} \square \delta g^{\mu \nu}\right) \\
& =R_{\mu \nu} \delta g^{\mu \nu}-K_{\mu \nu} \delta g^{\mu \nu} .
\end{aligned}
$$

The last equation (21) is proved in the following way

$$
\begin{aligned}
\delta \nabla_{\mu} \nabla_{\nu} \psi & =\delta\left(\partial_{\mu} \nabla_{\nu} \psi-\Gamma_{\mu \nu}^{\lambda} \nabla_{\lambda} \psi\right) \\
& =\delta\left(\partial_{\mu \nu}^{2} \psi-\Gamma_{\mu \nu}^{\lambda} \partial_{\lambda} \psi\right) \\
& =\partial_{\mu \nu}^{2} \delta \psi-\Gamma_{\mu \nu}^{\lambda} \partial_{\lambda} \delta \psi-\partial_{\lambda} \psi \delta \Gamma_{\mu \nu}^{\lambda} \\
& =\nabla_{\mu} \nabla_{\nu} \delta \psi-\nabla_{\lambda} \psi \delta \Gamma_{\mu \nu}^{\lambda} .
\end{aligned}
$$

Lemma 3. Every scalar function $P(R)$ satisfies 


$$
\begin{gathered}
\int_{M} P g_{\mu \nu}\left(\square \delta g^{\mu \nu}\right) \sqrt{-g} \mathrm{~d}^{4} x=\int_{M} g_{\mu \nu}(\square P) \delta g^{\mu \nu} \sqrt{-g} \mathrm{~d}^{4} x \\
\int_{M} P \nabla_{\mu} \nabla_{\nu} \delta g^{\mu \nu} \sqrt{-g} \mathrm{~d}^{4} x=\int_{M} \nabla_{\mu} \nabla_{\nu} P \delta g^{\mu \nu} \sqrt{-g} \mathrm{~d}^{4} x \\
\int_{M} P K_{\mu \nu} \delta g^{\mu \nu} \sqrt{-g} \mathrm{~d}^{4} x=\int_{M} K_{\mu \nu} P \delta g^{\mu \nu} \sqrt{-g} \mathrm{~d}^{4} x .
\end{gathered}
$$

Proof. Equation (25) is proved by application of Stokes' theorem:

$$
\begin{aligned}
\int_{M} P g_{\mu \nu} \square \delta g^{\mu \nu} \sqrt{-g} \mathrm{~d}^{4} x & =\int_{M} P g_{\mu \nu} \nabla_{\alpha} \nabla^{\alpha} \delta g^{\mu \nu} \sqrt{-g} \mathrm{~d}^{4} x \\
& =-\int_{M} \nabla_{\alpha}\left(P g_{\mu \nu}\right) \nabla^{\alpha} \delta g^{\mu \nu} \sqrt{-g} \mathrm{~d}^{4} x \\
& =\int_{M} g_{\mu \nu} \nabla^{\alpha} \nabla_{\alpha} P \delta g^{\mu \nu} \sqrt{-g} \mathrm{~d}^{4} x \\
& =\int_{M} g_{\mu \nu} \square P \delta g^{\mu \nu} \sqrt{-g} \mathrm{~d}^{4} x .
\end{aligned}
$$

Let $N^{\mu}=P \nabla_{\nu} \delta g^{\mu \nu}-\nabla_{\nu} P \delta g^{\mu \nu}$, then $\nabla_{\mu} N^{\mu}$ can be written as

$$
\begin{aligned}
\nabla_{\mu} N^{\mu} & =\nabla_{\mu}\left(P \nabla_{\nu} \delta g^{\mu \nu}-\nabla_{\nu} P \delta g^{\mu \nu}\right) \\
& =\nabla_{\mu} P \nabla_{\nu} \delta g^{\mu \nu}+P \nabla_{\mu} \nabla_{\nu} \delta g^{\mu \nu}-\nabla_{\mu} \nabla_{\nu} P \delta g^{\mu \nu}-\nabla_{\nu} P \nabla_{\mu} \delta g^{\mu \nu} \\
& =P \nabla_{\mu} \nabla_{\nu} \delta g^{\mu \nu}-\nabla_{\mu} \nabla_{\nu} P \delta g^{\mu \nu} .
\end{aligned}
$$

Integration over $M$ yields $\int_{M} \nabla_{\mu} N^{\mu} \sqrt{-g} \mathrm{~d}^{4} x=\int_{\partial M} N^{\mu} n_{\mu} \mathrm{d} \partial M$, where $n_{\mu}$ is the unit normal to a hypersurface $\partial M$. As the restriction $\left.N^{\mu}\right|_{\partial M}$ vanish, the last integral vanish as well, which proves (26).

Equation (27) is a direct consequence of (25) and (26).

Lemma 4. Let $P(R)$ and $Q(R)$ be scalar functions. Then for all $n \in \mathbb{N}$

$$
\begin{aligned}
\int_{M} P \delta \square^{n} Q \sqrt{-g} \mathrm{~d}^{4} x & =\frac{1}{2} \sum_{l=0}^{n-1} \int_{M} S_{\mu \nu}\left(\square^{l} P, \square^{n-1-l} Q\right) \delta g^{\mu \nu} \sqrt{-g} \mathrm{~d}^{4} x \\
& +\int_{M} \square^{n} P \delta Q \sqrt{-g} \mathrm{~d}^{4} x .
\end{aligned}
$$

Proof. The definition of the $\square$ operator implies

$$
\begin{aligned}
I & =\int_{M} P \delta \square^{n} Q \sqrt{-g} \mathrm{~d}^{4} x=\int_{M} P \delta\left(g^{\mu \nu} \nabla_{\mu} \nabla_{\nu} \square^{n-1} Q\right) \sqrt{-g} \mathrm{~d}^{4} x \\
& =\int_{M} P\left(\nabla_{\mu} \nabla_{\nu} \square^{n-1} Q \delta g^{\mu \nu}+g^{\mu \nu} \delta \nabla_{\mu} \nabla_{\nu} \square^{n-1} Q\right) \sqrt{-g} \mathrm{~d}^{4} x \\
& =\int_{M} P\left(\nabla_{\mu} \nabla_{\nu} \square^{n-1} Q \delta g^{\mu \nu}+\square \delta \square^{n-1} Q-\nabla_{\lambda} \square^{n-1} Q g^{\mu \nu} \delta \Gamma_{\mu \nu}^{\lambda}\right) \sqrt{-g} \mathrm{~d}^{4} x .
\end{aligned}
$$


On the other hand, Lemma 1 yields

$$
\begin{aligned}
g^{\mu \nu} \delta \Gamma_{\mu \nu}^{\lambda} & =-\frac{1}{2} g^{\mu \nu}\left(g_{\nu \alpha} \nabla_{\mu} \delta g^{\lambda \alpha}+g_{\mu \alpha} \nabla_{\nu} \delta g^{\lambda \alpha}-g_{\mu \alpha} g_{\nu \beta} \nabla^{\lambda} \delta g^{\alpha \beta}\right) \\
& =-\frac{1}{2}\left(\delta_{\alpha}^{\mu} \nabla_{\mu} \delta g^{\lambda \alpha}+\delta_{\alpha}^{\nu} \nabla_{\nu} \delta g^{\lambda \alpha}-\delta_{\alpha}^{\nu} g_{\nu \beta} \nabla^{\lambda} \delta g^{\alpha \beta}\right) \\
& =-\frac{1}{2}\left(2 \nabla_{\mu} \delta g^{\lambda \mu}-g_{\mu \nu} \nabla^{\lambda} \delta g^{\mu \nu}\right)
\end{aligned}
$$

Moreover, from the equation (31) and Stokes' theorem we get

$$
\begin{aligned}
I & =\int_{M} P\left(\nabla_{\mu} \nabla_{\nu} \square^{n-1} Q \delta g^{\mu \nu}+\square \delta \square^{n-1} Q\right. \\
& \left.+\frac{1}{2} \nabla_{\lambda} \square^{n-1} Q\left(2 \nabla_{\mu} \delta g^{\lambda \mu}-g_{\mu \nu} \nabla^{\lambda} \delta g^{\mu \nu}\right)\right) \sqrt{-g} \mathrm{~d}^{4} x \\
& =\int_{M} P \nabla_{\mu} \nabla_{\nu} \square^{n-1} Q \delta g^{\mu \nu} \sqrt{-g} \mathrm{~d}^{4} x+\int_{M} P \square \delta \square^{n-1} Q \sqrt{-g} \mathrm{~d}^{4} x \\
& -\int_{M} \nabla_{\mu}\left(P \nabla_{\lambda} \square^{n-1} Q\right) \delta g^{\lambda \mu} \sqrt{-g} \mathrm{~d}^{4} x \\
& -\frac{1}{2} \int_{M} g_{\mu \nu} \nabla^{\lambda}\left(P \nabla_{\lambda} \square^{n-1} Q\right) \delta g^{\mu \nu} \sqrt{-g} \mathrm{~d}^{4} x \\
& =\int_{M} P \square \delta \square^{n-1} Q \sqrt{-g} \mathrm{~d}^{4} x-\int_{M} \nabla_{\mu} P \nabla_{\nu} \square^{n-1} Q \delta g^{\mu \nu} \sqrt{-g} \mathrm{~d}^{4} x \\
& -\frac{1}{2} \int_{M} g_{\mu \nu}\left(\nabla^{\lambda} P \nabla_{\lambda} \square^{n-1} Q+P \square^{n-1} Q\right) \delta g^{\mu \nu} \sqrt{-g} \mathrm{~d}^{4} x \\
& =\int_{M} P \square \delta \square^{n-1} Q \sqrt{-g} \mathrm{~d}^{4} x+\frac{1}{2} \int_{M} S_{\mu \nu}\left(P, \square^{n-1} Q\right) \delta g^{\mu \nu} \sqrt{-g} \mathrm{~d}^{4} x .
\end{aligned}
$$

Partial integration in the first term of the previous formula yields

$$
I=\int_{M} \square P \delta \square^{n-1} Q \sqrt{-g} \mathrm{~d}^{4} x+\frac{1}{2} \int_{M} S_{\mu \nu}\left(P, \square^{n-1} Q\right) \delta g^{\mu \nu} \sqrt{-g} \mathrm{~d}^{4} x .
$$

after $n-1$ more steps

$$
I=\int_{M}\left(\square^{n} P \delta Q+\frac{1}{2} \sum_{l=0}^{n-1} S_{\mu \nu}\left(\square^{l} P, \square^{n-1-l} Q\right) \delta g^{\mu \nu}\right) \sqrt{-g} \mathrm{~d}^{4} x
$$

Theorem 1. Let $P$ and $Q$ be scalar functions of scalar curvature, then 


$$
\begin{aligned}
\int_{M} P \delta(\sqrt{-g}) \mathrm{d}^{4} x & =-\frac{1}{2} \int_{M} g_{\mu \nu} P \delta g^{\mu \nu} \sqrt{-g} \mathrm{~d}^{4} x \\
\int_{M} P \delta R \sqrt{-g} \mathrm{~d}^{4} x & =\int_{M}\left(R_{\mu \nu} P-K_{\mu \nu} P\right) \delta g^{\mu \nu} \sqrt{-g} \mathrm{~d}^{4} x \\
\int_{M} P \delta(\mathcal{F}(\square) Q) \sqrt{-g} \mathrm{~d}^{4} x & =\int_{M}\left(R_{\mu \nu}-K_{\mu \nu}\right)\left(Q^{\prime} \mathcal{F}(\square) P\right) \delta g^{\mu \nu} \sqrt{-g} \mathrm{~d}^{4} x \\
& +\frac{1}{2} \sum_{n=1}^{\infty} f_{n} \sum_{l=0}^{n-1} \int_{M} S_{\mu \nu}\left(\square^{l} P, \square^{n-1-l} Q\right) \delta g^{\mu \nu} \sqrt{-g} \mathrm{~d}^{4} x
\end{aligned}
$$

where $S_{\mu \nu}(A, B)=g_{\mu \nu} \nabla^{\alpha} A \nabla_{\alpha} B+g_{\mu \nu} A \square B-2 \nabla_{\mu} A \nabla_{\nu} B$.

Proof. Equation (36) is a consequence of (44).

From Lemmas 2 and 3 we get

$$
\begin{aligned}
\int_{M} P \delta R \sqrt{-g} \mathrm{~d}^{4} x & =\int_{M}\left(R_{\mu \nu} P \delta g^{\mu \nu}-P K_{\mu \nu} \delta g^{\mu \nu}\right) \sqrt{-g} \mathrm{~d}^{4} x \\
& =\int_{M}\left(R_{\mu \nu} P-K_{\mu \nu} P\right) \delta g^{\mu \nu} \sqrt{-g} \mathrm{~d}^{4} x .
\end{aligned}
$$

To prove (38) let us introduce the following notation

$$
J_{n}=\int_{M} P \delta\left(\square^{n} Q\right) \sqrt{-g} \mathrm{~d}^{4} x .
$$

Then,

$$
\int_{M} P \delta(\mathcal{F}(\square) Q) \sqrt{-g} \mathrm{~d}^{4} x=\sum_{n=0}^{\infty} f_{n} J_{n} .
$$

The integral $J_{0}$ is calculated by applying (37), i.e.

$$
J_{0}=\int_{M}\left(R_{\mu \nu} Q^{\prime} P-K_{\mu \nu} Q^{\prime} P\right) \delta g^{\mu \nu} \sqrt{-g} \mathrm{~d}^{4} x .
$$

For $n>0$ integral $J_{n}$ is calculated by applying Lemma 4 .

$$
J_{n}=\int_{M} \square^{n} P \delta Q \sqrt{-g} \mathrm{~d}^{4} x+\frac{1}{2} \sum_{l=0}^{n-1} \int_{M} S_{\mu \nu}\left(\square^{l} P, \square^{n-1-l} Q\right) \delta g^{\mu \nu} \sqrt{-g} \mathrm{~d}^{4} x .
$$

Using (37) in the first term we obtain 


$$
\begin{aligned}
J_{n} & =\int_{M}\left(R_{\mu \nu} Q^{\prime} \square^{n} P-K_{\mu \nu}\left(Q^{\prime} \square^{n} P\right)\right) \delta g^{\mu \nu} \sqrt{-g} \mathrm{~d}^{4} x \\
& +\frac{1}{2} \sum_{l=0}^{n-1} \int_{M} S_{\mu \nu}\left(\square^{l} P, \square^{n-1-l} Q\right) \delta g^{\mu \nu} \sqrt{-g} \mathrm{~d}^{4} x .
\end{aligned}
$$

Summation over $n$ yields the final result

$$
\begin{aligned}
I & =\int_{M} P \delta(\mathcal{F}(\square) Q) \sqrt{-g} \mathrm{~d}^{4} x=\sum_{n=0}^{\infty} f_{n} J_{n} \\
& =\sum_{n=0}^{\infty} f_{n} \int_{M}\left(R_{\mu \nu} Q^{\prime} \square^{n} P-K_{\mu \nu}\left(Q^{\prime} \square^{n} P\right)\right) \delta g^{\mu \nu} \sqrt{-g} \mathrm{~d}^{4} x \\
& +\frac{1}{2} \sum_{n=1}^{\infty} \sum_{l=0}^{n-1} f_{n} \int_{M} S_{\mu \nu}\left(\square^{l} P, \square^{n-1-l} Q\right) \delta g^{\mu \nu} \sqrt{-g} \mathrm{~d}^{4} x \\
& =\int_{M}\left(R_{\mu \nu} Q^{\prime} \mathcal{F}(\square) P-K_{\mu \nu}\left(Q^{\prime} \mathcal{F}(\square) P\right)\right) \delta g^{\mu \nu} \sqrt{-g} \mathrm{~d}^{4} x \\
& +\frac{1}{2} \sum_{n=1}^{\infty} \sum_{l=0}^{n-1} f_{n} \int_{M} S_{\mu \nu}\left(\square^{l} P, \square^{n-1-l} Q\right) \delta g^{\mu \nu} \sqrt{-g} \mathrm{~d}^{4} x .
\end{aligned}
$$

\section{Equations of motion}

Let us return to action (11). In order to calculate $\delta S$ we introduce the following auxiliary actions

$$
\begin{aligned}
S_{0} & =\int_{M}(R-2 \Lambda) \sqrt{-g} \mathrm{~d}^{4} x, \\
S_{1} & =\int_{M} P(R) \mathcal{F}(\square) Q(R) \sqrt{-g} \mathrm{~d}^{4} x .
\end{aligned}
$$

Action $S_{0}$ is Einstein-Hilbert action and its variation is

$$
\delta S_{0}=\int_{M}\left(G_{\mu \nu}+\Lambda g_{\mu \nu}\right) \delta g^{\mu \nu} \sqrt{-g} \mathrm{~d}^{4} x .
$$

Lemma 5. Variation of the action $S_{1}$ is 


$$
\begin{aligned}
\delta S_{1} & =-\frac{1}{2} \int_{M} g_{\mu \nu} P(R) \mathcal{F}(\square) Q(R) \delta g^{\mu \nu} \sqrt{-g} \mathrm{~d}^{4} x \\
& +\int_{M}\left(R_{\mu \nu} W-K_{\mu \nu} W\right) \delta g^{\mu \nu} \sqrt{-g} \mathrm{~d}^{4} x \\
& +\frac{1}{2} \sum_{n=1}^{\infty} f_{n} \sum_{l=0}^{n-1} \int_{M} S_{\mu \nu}\left(\square^{l} P(R), \square^{n-1-l} Q(R)\right) \delta g^{\mu \nu} \sqrt{-g} \mathrm{~d}^{4} x,
\end{aligned}
$$

where $W=P^{\prime}(R) \mathcal{F}(\square) Q(R)+Q^{\prime}(R) \mathcal{F}(\square) P(R)$.

Proof. Variation $\delta S_{1}$ is equal to

$$
\begin{aligned}
\delta S_{1} & =\int_{M}(P(R) \mathcal{F}(\square) Q(R) \delta(\sqrt{-g})+\delta P(R) \mathcal{F}(\square) Q(R) \sqrt{-g} \\
& +P(R) \delta(\mathcal{F}(\square) Q(R)) \sqrt{-g}) \mathrm{d}^{4} x .
\end{aligned}
$$

All the terms in the previous formula are obtained by Theorem 1. In particular (36) yields

$\int_{M} P(R) \mathcal{F}(\square) Q(R) \delta(\sqrt{-g}) \mathrm{d}^{4} x=-\frac{1}{2} \int_{M} g_{\mu \nu} P(R) \mathcal{F}(\square) Q(R) \delta g^{\mu \nu} \sqrt{-g} \mathrm{~d}^{4} x$.

Also, from equation (37) we get

$$
\begin{aligned}
& \int_{M} \delta(P(R)) \mathcal{F}(\square) Q(R) \sqrt{-g} \mathrm{~d}^{4} x=\int_{M} P^{\prime}(R) \delta R \mathcal{F}(\square) Q(R) \sqrt{-g} \mathrm{~d}^{4} x \\
& =\int_{M}\left(R_{\mu \nu} P^{\prime}(R) \mathcal{F}(\square) Q(R)-K_{\mu \nu}\left(P^{\prime}(R) \mathcal{F}(\square) Q(R)\right)\right) \delta g^{\mu \nu} \sqrt{-g} \mathrm{~d}^{4} x .
\end{aligned}
$$

The last term is calculated by (38).

$$
\begin{array}{rl}
\int_{M} & P(R) \delta(\mathcal{F}(\square) Q(R)) \sqrt{-g} \mathrm{~d}^{4} x \\
& =\int_{M}\left(R_{\mu \nu} Q^{\prime}(R) \mathcal{F}(\square) P(R)-K_{\mu \nu}\left(Q^{\prime}(R) \mathcal{F}(\square) P(R)\right)\right) \delta g^{\mu \nu} \sqrt{-g} \mathrm{~d}^{4} x \\
& +\sum_{n=1}^{\infty} f_{n} \sum_{l=0}^{n-1} \int_{M} S_{\mu \nu}\left(\square^{l} P(R), \square^{n-1-l} Q(R)\right) \delta g^{\mu \nu} \sqrt{-g} \mathrm{~d}^{4} x .
\end{array}
$$

Adding equations (51), (52) and (53) together proves the Lemma.

Theorem 2. Variation of the action (1) is equal to zero iff 
$\hat{G}_{\mu \nu}=G_{\mu \nu}+\Lambda g_{\mu \nu}-\frac{1}{2} g_{\mu \nu} P(R) \mathcal{F}(\square) Q(R)+\left(R_{\mu \nu} W-K_{\mu \nu} W\right)+\frac{1}{2} \Omega_{\mu \nu}=0$,

where

$$
\begin{gathered}
W=P^{\prime}(R) \mathcal{F}(\square) Q(R)+Q^{\prime}(R) \mathcal{F}(\square) P(R), \\
\Omega_{\mu \nu}=\sum_{n=1}^{\infty} f_{n} \sum_{l=0}^{n-1} S_{\mu \nu}\left(\square^{l} P(R), \square^{n-1-l} Q(R)\right) .
\end{gathered}
$$

Proof. The proof of Theorem 2 is evident from the Lemma 5 and Theorem 1.

\section{Second variation of the action}

In this section we set $h_{\mu \nu}=\delta g_{\mu \nu}$. From Lemma 2 we see that $h^{\mu \nu}=-\delta g^{\mu \nu}$. Also let $h=g^{\mu \nu} h_{\mu \nu}$ be the trace of $h_{\mu \nu}$.

Operator $\delta \square$ is defined by $(\delta \square) V=\delta(\square V)-\square \delta V$. Then we can prove the following Lemma

Lemma 6. Let $U, V$ be scalar functions. Then

$$
\begin{aligned}
(\delta \square) V & =-h^{\mu \nu} \nabla_{\mu} \nabla_{\nu} V-\nabla^{\mu} h_{\mu}^{\lambda} \nabla_{\lambda} V+\frac{1}{2} \nabla^{\lambda} h \nabla_{\lambda} V, \\
\int_{M} U(\delta \square) V \sqrt{-g} \mathrm{~d}^{4} x & =\frac{1}{2} \int_{M} S_{\mu \nu}(U, V) \delta g^{\mu \nu} \sqrt{-g} \mathrm{~d}^{4} x .
\end{aligned}
$$

Proof. For the first part, start with

$$
\begin{aligned}
(\delta \square) V & =\delta(\square V)-\square \delta V \\
& =\delta\left(g^{\mu \nu} \nabla_{\mu} \nabla_{\nu} V\right)-\square \delta V \\
& =-h^{\mu \nu} \nabla_{\mu} \nabla_{\nu} V-g^{\mu \nu} \delta \Gamma_{\mu \nu}^{\lambda} \nabla_{\lambda} V \\
& =-h^{\mu \nu} \nabla_{\mu} \nabla_{\nu} V-\frac{1}{2} g^{\mu \nu}\left(\nabla_{\mu} h_{\nu}^{\lambda}+\nabla_{\nu} h_{\mu}^{\lambda}-\nabla^{\lambda} h_{\mu \nu}\right) \nabla_{\lambda} V \\
& =-h^{\mu \nu} \nabla_{\mu} \nabla_{\nu} V-\nabla^{\mu} h_{\mu}^{\lambda} \nabla_{\lambda} V+\frac{1}{2} \nabla^{\lambda} h \nabla_{\lambda} V .
\end{aligned}
$$

The second part of the Lemma is proved by 


$$
\begin{aligned}
& \int_{M} U(\delta \square) V \sqrt{-g} \mathrm{~d}^{4} x \\
& =\int_{M} U\left(-h^{\mu \nu} \nabla_{\mu} \nabla_{\nu} V-\nabla^{\mu} h_{\mu}^{\lambda} \nabla_{\lambda} V+\frac{1}{2} \nabla^{\lambda} h \nabla_{\lambda} V\right) \sqrt{-g} \mathrm{~d}^{4} x \\
& =-\int_{M}\left(U \nabla_{\mu} \nabla_{\nu} V-\nabla_{\mu}\left(U \nabla_{\nu} V\right)+\frac{1}{2} g_{\mu \nu} \nabla^{\lambda}\left(U \nabla_{\lambda} V\right)\right) h^{\mu \nu} \sqrt{-g} \mathrm{~d}^{4} x \\
& =\frac{1}{2} \int_{M}\left(-2 \nabla_{\mu} U \nabla_{\nu} V+g_{\mu \nu} \nabla^{\lambda} U \nabla_{\lambda} V+g_{\mu \nu} U \square V\right) \delta g^{\mu \nu} \sqrt{-g} \mathrm{~d}^{4} x \\
& =\frac{1}{2} \int_{M} S_{\mu \nu}(U, V) \delta g^{\mu \nu} \sqrt{-g} \mathrm{~d}^{4} x .
\end{aligned}
$$

In the next lemma we find the variation of $\mathcal{F}(\square)$.

Lemma 7. Let $U, V$ be scalar functions. Then,

$$
\int_{M} U \delta(\mathcal{F}(\square)) V \sqrt{-g} \mathrm{~d}^{4} x=\sum_{n=1}^{\infty} f_{n} \sum_{l=0}^{n-1} \int_{M} S_{\mu \nu}\left(\square^{l} U, \square^{n-1-l} V\right) \delta g^{\mu \nu} \sqrt{-g} \mathrm{~d}^{4} x .
$$

Proof. Note that $\delta \square^{n}=\sum_{l=0}^{n-1} \square^{l}(\delta \square) \square^{n-1-l}$ for $n>0$ and $\delta \square^{0}=\delta \mathrm{Id}=0$. Therefore summation over $n$ and integration yields

$$
\begin{aligned}
& \int_{M} U \delta(\mathcal{F}(\square)) V \sqrt{-g} \mathrm{~d}^{4} x=\sum_{n=1}^{\infty} f_{n} \sum_{l=0}^{n-1} \int_{M} U \square^{l}(\delta \square) \square^{n-1-l} V \sqrt{-g} \mathrm{~d}^{4} x \\
& =\sum_{n=1}^{\infty} f_{n} \sum_{l=0}^{n-1} \int_{M} \square^{l} U(\delta \square) \square^{n-1-l} V \sqrt{-g} \mathrm{~d}^{4} x \\
& =\sum_{n=1}^{\infty} f_{n} \sum_{l=0}^{n-1} \int_{M} S_{\mu \nu}\left(\square^{l} U, \square^{n-1-l} V\right) \delta g^{\mu \nu} \sqrt{-g} \mathrm{~d}^{4} x .
\end{aligned}
$$

Lemma 8. Let $U$ be scalar function. Then,

$$
\begin{gathered}
\int_{M} U \delta W \sqrt{-g} \mathrm{~d}^{4} x=\int_{M}\left(R_{\mu \nu} Y-K_{\mu \nu} Y+\frac{1}{2} \Psi_{\mu \nu}\right) \delta g^{\mu \nu} \sqrt{-g} \mathrm{~d}^{4} x, \\
Y=U\left(P^{\prime \prime} \mathcal{F}(\square) Q+Q^{\prime \prime} \mathcal{F}(\square) P\right)+\left(P^{\prime} \mathcal{F}(\square)\left(Q^{\prime} U\right)+Q^{\prime} \mathcal{F}(\square)\left(P^{\prime} U\right)\right), \\
\Psi_{\mu \nu}=\sum_{n=1}^{+\infty} f_{n} \sum_{l=0}^{n-1}\left(S_{\mu \nu}\left(\square^{l}\left(P^{\prime} U\right), \square^{n-1-l} Q\right)+S_{\mu \nu}\left(\square^{l}\left(Q^{\prime} U\right), \square^{n-1-l} P\right)\right) .
\end{gathered}
$$

Proof. Since $W=P^{\prime} \mathcal{F}(\square) Q+Q^{\prime} \mathcal{F}(\square) P$ the variation $\delta W$ is written as 


$$
\begin{aligned}
\delta W & =P^{\prime \prime} \mathcal{F}(\square) Q \delta R+P^{\prime} \delta(\mathcal{F}(\square)) Q+P^{\prime} \mathcal{F}(\square)\left(Q^{\prime} \delta R\right) \\
& +Q^{\prime \prime} \mathcal{F}(\square) P \delta R+Q^{\prime} \delta(\mathcal{F}(\square)) P+Q^{\prime} \mathcal{F}(\square)\left(P^{\prime} \delta R\right) .
\end{aligned}
$$

Integration of the second and fifth term in this sum is done by using Lemma 7. The remaining four terms are obtained by Theorem 1

Lemma 9. Let $A, B$ be scalar functions. Then,

$$
\begin{aligned}
& \int_{M} S_{\mu \nu}(\delta A, B) \delta g^{\mu \nu} \sqrt{-g} \mathrm{~d}^{4} x=\int \sigma_{1}(B) \delta A \sqrt{-g} \mathrm{~d}^{4} x, \\
& \int_{M} S_{\mu \nu}(A, \delta B) \delta g^{\mu \nu} \sqrt{-g} \mathrm{~d}^{4} x=\int \sigma_{2}(A) \delta B \sqrt{-g} \mathrm{~d}^{4} x,
\end{aligned}
$$

where

$$
\begin{aligned}
& \sigma_{1}(B)=\nabla^{\lambda} h \nabla_{\lambda} B-2 \nabla_{\mu} h^{\mu \nu} \nabla_{\nu} B-2 h^{\mu \nu} \nabla_{\mu} \nabla_{\nu} B, \\
& \sigma_{2}(A)=-\nabla^{\lambda} h \nabla_{\lambda} A-A \square h-2 \nabla_{\nu} h^{\mu \nu} \nabla_{\mu} A-2 h^{\mu \nu} \nabla_{\mu} \nabla_{\nu} A .
\end{aligned}
$$

Proof. To prove the first equation recall the definition of $S_{\mu \nu}(A, B)$

$$
\begin{aligned}
& \int_{M} S_{\mu \nu}(\delta A, B) \delta g^{\mu \nu} \sqrt{-g} \mathrm{~d}^{4} x \\
& =\int_{M}\left(g_{\mu \nu} \nabla^{\alpha} \delta A \nabla_{\alpha} B+g_{\mu \nu} \delta A \square B-2 \nabla_{\mu} \delta A \nabla_{\nu} B\right) \delta g^{\mu \nu} \sqrt{-g} \mathrm{~d}^{4} x \\
& =\int_{M}\left(-h \nabla^{\alpha} \delta A \nabla_{\alpha} B-h \delta A \square B+2 h^{\mu \nu} \nabla_{\mu} \delta A \nabla_{\nu} B\right) \sqrt{-g} \mathrm{~d}^{4} x \\
& =\int_{M}\left(\nabla^{\alpha}\left(h \nabla_{\alpha} B\right)-h \square B-2 \nabla_{\mu}\left(h^{\mu \nu} \nabla_{\nu} B\right)\right) \delta A \sqrt{-g} \mathrm{~d}^{4} x \\
& =\int_{M}\left(\nabla^{\alpha} h \nabla_{\alpha} B-2 \nabla_{\mu} h^{\mu \nu} \nabla_{\nu} B-2 h^{\mu \nu} \nabla_{\mu} \nabla_{\nu} B\right) \delta A \sqrt{-g} \mathrm{~d}^{4} x \\
& =\int_{M} \sigma_{1}(B) \delta A \sqrt{-g} \mathrm{~d}^{4} x .
\end{aligned}
$$

The proof of the second equation is similar.

Lemma 10. Let $\Omega_{\mu \nu}=\sum_{n=1}^{\infty} f_{n} \sum_{l=0}^{n-1} S_{\mu \nu}\left(\square^{l} P(R), \square^{n-1-l} Q(R)\right)$. Then, 


$$
\begin{aligned}
& \int_{M} \delta \Omega_{\mu \nu} \delta g^{\mu \nu} \sqrt{-g} \mathrm{~d}^{4} x \\
& =\int_{M} \sum_{n=1}^{+\infty} f_{n} \sum_{l=0}^{n-1}\left(h_{\mu \nu} \nabla^{\lambda} \square^{l} P \nabla_{\lambda} \square^{n-1-l} Q+h \nabla_{\mu} \square^{l} P \nabla_{\nu} \square^{n-1-l} Q\right. \\
& +h_{\mu \nu} \square^{l} P \square^{n-l} Q-\frac{1}{2} S_{\mu \nu}\left(h \square^{l} P, \square^{n-1-l} Q\right) \\
& +R_{\mu \nu} P^{\prime} \square^{l}\left(\sigma_{1}\left(\square^{n-1-l} Q\right)\right)-K_{\mu \nu}\left(P^{\prime} \square^{l}\left(\sigma_{1}\left(\square^{n-1-l} Q\right)\right)\right) \\
& \left.+R_{\mu \nu} Q^{\prime} \square^{l}\left(\sigma_{2}\left(\square^{n-1-l} P\right)\right)-K_{\mu \nu}\left(Q^{\prime} \square^{l}\left(\sigma_{2}\left(\square^{n-1-l} P\right)\right)\right)\right) \delta g^{\mu \nu} \sqrt{-g} \mathrm{~d}^{4} x \\
& +\frac{1}{2} \int_{M} \sum_{n=1}^{+\infty} f_{n} \sum_{l=1}^{n-1} \sum_{m=0}^{l-1}\left(S_{\mu \nu}\left(\square^{m}\left(\sigma_{1}\left(\square^{n-1-l} Q\right)\right), \square^{l-m-1} P\right)\right. \\
& \left.+S_{\mu \nu}\left(\square^{m}\left(\sigma_{2}\left(\square^{n-1-l} P\right)\right), \square^{l-m-1} Q\right)\right) \delta g^{\mu \nu} \sqrt{-g} \mathrm{~d}^{4} x .
\end{aligned}
$$

Proof. Note that

$$
\delta \Omega_{\mu \nu}=\sum_{n=1}^{\infty} f_{n} \sum_{l=0}^{n-1} \delta S_{\mu \nu}\left(\square^{l} P(R), \square^{n-1-l} Q(R)\right) .
$$

Moreover,

$$
\begin{aligned}
& \int_{M} \delta S_{\mu \nu}(A, B) \delta g^{\mu \nu} \sqrt{-g} \mathrm{~d}^{4} x=\int_{M}\left(S_{\mu \nu}(\delta A, B)+S_{\mu \nu}(A, \delta B)\right) \delta g^{\mu \nu} \sqrt{-g} \mathrm{~d}^{4} x \\
& +\int_{M}\left(h_{\mu \nu} \nabla^{\lambda} A \nabla_{\lambda} B+h \nabla_{\mu} A \nabla_{\nu} B\right. \\
& \left.+h_{\mu \nu} A \square B-\frac{1}{2} S_{\mu \nu}(h A, B)\right) \delta g^{\mu \nu} \sqrt{-g} \mathrm{~d}^{4} x .
\end{aligned}
$$

Using this formula for each term in $\delta \Omega_{\mu \nu}$ yields the result of the Lemma.

Theorem 3. The second variation of the action (11) is given by

$$
\delta^{2} S=\frac{1}{16 \pi G} \int_{M}\left(U_{\mu \nu}+R_{\mu \nu} X-K_{\mu \nu} X+\frac{1}{2} \chi_{\mu \nu}+\frac{1}{4} \Theta_{\mu \nu}\right) \delta g^{\mu \nu} \sqrt{-g} \mathrm{~d}^{4} x
$$

where 


$$
\begin{aligned}
U_{\mu \nu} & =-\frac{1}{2} h_{\mu \nu}(R-2 \Lambda+P \mathcal{F}(\square) Q)+\delta R_{\mu \nu}(W+1)+\delta \Gamma_{\mu \nu}^{\lambda} \nabla_{\lambda} W \\
& +h_{\mu \nu} \square W-\frac{1}{2} S_{\mu \nu}(h, W), \\
X & =\frac{1}{2}\left(h+P^{\prime} h \mathcal{F}(\square) Q+Q^{\prime} \mathcal{F}(\square)(P h)\right)+\left(\delta R\left(P^{\prime \prime} \mathcal{F}(\square) Q+Q^{\prime \prime} \mathcal{F}(\square) P\right)\right. \\
& \left.+\left(P^{\prime} \mathcal{F}(\square)\left(Q^{\prime} \delta R\right)+Q^{\prime} \mathcal{F}(\square)\left(P^{\prime} \delta R\right)\right)\right), \\
\chi_{\mu \nu}= & \frac{1}{2} \sum_{n=1}^{+\infty} f_{n} \sum_{l=0}^{n-1} S_{\mu \nu}\left(\square^{l}(P h), \square^{n-l-1} Q\right) \\
& -\sum_{n=1}^{+\infty} f_{n} \sum_{l=0}^{n-1}\left(S_{\mu \nu}\left(\square^{l}\left(P^{\prime} M\right), \square^{n-1-l} Q\right)+S_{\mu \nu}\left(\square^{l}\left(Q^{\prime} M\right), \square^{n-1-l} P\right)\right) \\
& +\sum_{n=1}^{+\infty} f_{n} \sum_{l=0}^{n-1}\left(h_{\mu \nu} \nabla^{\lambda} \square^{l} P \nabla_{\lambda} \square^{n-1-l} Q+h \nabla_{\mu} \square^{l} P \nabla_{\nu} \square^{n-1-l} Q\right. \\
& +h_{\mu \nu} \square^{l} P \square^{n-l} Q-\frac{1}{2} S_{\mu \nu}\left(h \square^{l} P, \square^{n-1-l} Q\right) \\
& \left.+\left(R_{\mu \nu}-K_{\mu \nu}\right)\left(P^{\prime} \square^{l}\left(\sigma_{1}\left(\square^{n-1-l} Q\right)\right)+Q^{\prime} \square^{l}\left(\sigma_{2}\left(\square^{n-1-l} P\right)\right)\right)\right)
\end{aligned}
$$

and

$$
\begin{aligned}
\Theta_{\mu \nu} & =\sum_{n=1}^{+\infty} f_{n} \sum_{l=1}^{n-1} \sum_{m=0}^{l-1}\left(S_{\mu \nu}\left(\square^{m}\left(\sigma_{1}\left(\square^{n-1-l} Q\right)\right), \square^{l-m-1} P\right)\right. \\
& \left.+S_{\mu \nu}\left(\square^{m}\left(\sigma_{2}\left(\square^{n-1-l} P\right)\right), \square^{l-m-1} Q\right)\right), \\
\sigma_{1}(B) & =\nabla^{\lambda} h \nabla_{\lambda} B-2 \nabla_{\mu} h^{\mu \nu} \nabla_{\nu} B-2 h^{\mu \nu} \nabla_{\mu} \nabla_{\nu} B, \\
\sigma_{2}(A) & =-\nabla^{\lambda} h \nabla_{\lambda} A-A \square h-2 \nabla_{\nu} h^{\mu \nu} \nabla_{\mu} A-2 h^{\mu \nu} \nabla_{\mu} \nabla_{\nu} A .
\end{aligned}
$$

Proof. In the pervious section we calculated the first variation of the action (11)

$$
\delta S=\frac{1}{16 \pi G} \int_{M} \hat{G}_{\mu \nu} \delta g^{\mu \nu} \sqrt{-g} \mathrm{~d}^{4} x .
$$

Moreover the second variation $\delta^{2} S$ is

$$
\delta^{2} S=\frac{1}{16 \pi G} \int_{M}\left(\delta \hat{G}_{\mu \nu} \delta g^{\mu \nu}+\hat{G}_{\mu \nu} \delta^{2} g^{\mu \nu}-\frac{1}{2} g_{\alpha \beta} \delta g^{\alpha \beta} \hat{G}_{\mu \nu} \delta g^{\mu \nu}\right) \sqrt{-g} \mathrm{~d}^{4} x .
$$

At the beginning note that 


$$
\begin{aligned}
& \int_{M} \delta\left(G_{\mu \nu}+\Lambda g_{\mu \nu}\right) \delta g^{\mu \nu} \sqrt{-g} \mathrm{~d}^{4} x \\
& =\int_{M}\left(\delta R_{\mu \nu}-\frac{1}{2}(R-2 \Lambda) h_{\mu \nu}+\frac{1}{2} R_{\mu \nu} h-\frac{1}{2} K_{\mu \nu} h\right) \delta g^{\mu \nu} \sqrt{-g} \mathrm{~d}^{4} x .
\end{aligned}
$$

The next term is calculated by using Lemma 7

$$
\begin{aligned}
& \int_{M} \delta\left(g_{\mu \nu} P \mathcal{F}(\square) Q\right) \delta g^{\mu \nu} \sqrt{-g} \mathrm{~d}^{4} x \\
= & \int_{M} h_{\mu \nu} P \mathcal{F}(\square) Q \delta g^{\mu \nu} \sqrt{-g} \mathrm{~d}^{4} x+\int g_{\mu \nu} P \delta(\mathcal{F}(\square)) Q \delta g^{\mu \nu} \sqrt{-g} \mathrm{~d}^{4} x \\
- & \int_{M}\left(P^{\prime} h \mathcal{F}(\square) Q+Q^{\prime} \mathcal{F}(\square)(P h)\right) \delta R \sqrt{-g} \mathrm{~d}^{4} x \\
= & \int_{M} h_{\mu \nu} P \mathcal{F}(\square) Q \delta g^{\mu \nu} \sqrt{-g} \mathrm{~d}^{4} x \\
- & \frac{1}{2} \sum_{n=1}^{+\infty} f_{n} \sum_{l=0}^{n-1} \int S_{\mu \nu}\left(\square^{l}(P h), \square^{n-l-1} Q\right) \delta g^{\mu \nu} \sqrt{-g} \mathrm{~d}^{4} x \\
- & \int_{M} R_{\mu \nu}\left(P^{\prime} h \mathcal{F}(\square) Q+Q^{\prime} \mathcal{F}(\square)(P h)\right) \delta g^{\mu \nu} \sqrt{-g} \mathrm{~d}^{4} x \\
+ & \int K_{\mu \nu}\left(P^{\prime} h \mathcal{F}(\square) Q+Q^{\prime} \mathcal{F}(\square)(P h)\right) \delta g^{\mu \nu} \sqrt{-g} \mathrm{~d}^{4} x
\end{aligned}
$$

The third term is $\int_{M} \delta\left(R_{\mu \nu} W\right) \delta g^{\mu \nu} \sqrt{-g} \mathrm{~d}^{4} x$ and it is equal to

$$
\begin{aligned}
& \int_{M} \delta\left(R_{\mu \nu} W\right) \delta g^{\mu \nu} \sqrt{-g} \mathrm{~d}^{4} x \\
= & \int_{M}\left(W \delta R_{\mu \nu}+R_{\mu \nu} \delta W\right) \delta g^{\mu \nu} \sqrt{-g} \mathrm{~d}^{4} x \\
= & -\frac{1}{2} \int_{M}\left(\square h_{\mu \nu}+\nabla_{\mu} \nabla_{\nu} h-2 \nabla_{\lambda} \nabla_{\mu} h_{\nu}^{\lambda}\right) W \delta g^{\mu \nu} \sqrt{-g} \mathrm{~d}^{4} x \\
& +\int_{M} R_{\mu \nu} \delta W \delta g^{\mu \nu} \sqrt{-g} \mathrm{~d}^{4} x .
\end{aligned}
$$

The last integral of the above formula is obtained by Lemma 8, Similarly, we obtain 


$$
\begin{aligned}
& \int_{M} \delta\left(K_{\mu \nu} W\right) \delta g^{\mu \nu} \sqrt{-g} \mathrm{~d}^{4} x \\
= & \int_{M} K_{\mu \nu} \delta W \delta g^{\mu \nu} \sqrt{-g} \mathrm{~d}^{4} x \\
& +\int\left(\delta \Gamma_{\mu \nu}^{\lambda} \nabla_{\lambda} W+h_{\mu \nu} \square W+g_{\mu \nu}(\delta \square) W\right) h^{\mu \nu} \sqrt{-g} \mathrm{~d}^{4} x \\
= & \int_{M} \delta W K_{\mu \nu} \delta g^{\mu \nu} \sqrt{-g} \mathrm{~d}^{4} x \\
& -\int\left(\delta \Gamma_{\mu \nu}^{\lambda} \nabla_{\lambda} W+h_{\mu \nu} \square W-\frac{1}{2} S_{\mu \nu}(h, W)\right) \delta g^{\mu \nu} \sqrt{-g} \mathrm{~d}^{4} x .
\end{aligned}
$$
10.

At the end the last term $\int_{M} \delta \Omega_{\mu \nu} \delta g^{\mu \nu} \sqrt{-g} \mathrm{~d}^{4} x$ is calculated in Lemma

\section{Perturbations}

\subsection{Background}

In this section we start with $F R W$ metric, which for $k=0$ can be written as

$$
d s^{2}=-d t^{2}+a(t)^{2}\left(\mathrm{~d} x^{2}+\mathrm{d} y^{2}+\mathrm{d} z^{2}\right) .
$$

Some relevant background quantities are

$$
R=12 H^{2}+6 \dot{H}, \Gamma_{i j}^{0}=H g_{i j}, \Gamma_{j 0}^{i}=H \delta_{j}^{i}, \square=-\partial_{t}^{2}-3 H \partial_{t} .
$$

For perturbations it is useful to employ the canonical ADM $(1+3)$ decomposition and introduce the conformal time $\tau$ such that

$$
a \mathrm{~d} \tau=d t
$$

Then the flat FRW metric (110) transforms to

$$
d s^{2}=a(\tau)^{2}\left(-d \tau^{2}+\mathrm{d} x^{2}+\mathrm{d} y^{2}+\mathrm{d} z^{2}\right) .
$$

\subsection{Perturbations}

The metric perturbations (see 29]) can be divided into three types: scalar, vector and tensor perturbations. The component $h_{00}$ is invariant under spatial rotations and translations and therefore 


$$
h_{00}=2 a(\tau)^{2} \phi .
$$

The components $h_{0 i}$ are the sum of a spatial gradient of a function $B$ and divergence free vector $S_{i}$.

$$
h_{0 i}=a(\tau)^{2}\left(\partial_{i} B+S_{i}\right) .
$$

Similarly, components $h_{i j}$, which transform as a tensor under 3-rotations are written as

$$
h_{i j}=a(\tau)^{2}\left(2 \psi \delta_{i j}+2 \partial_{i j}^{2} E+\partial_{i} F_{j}+\partial_{j} F_{i}+\varphi_{i j}\right),
$$

where $\psi$ and $E$ are scalar functions, $F_{i}$ is a vector with zero divergence and 3 -tensor satisfies

$$
\varphi_{i}^{i}=0, \quad \partial_{i} \varphi_{j}^{i}=0 .
$$

Note that there are four scalar functions, two vectors with two independent components each and tensor $\varphi_{i j}$ has two independent components. Therefore, as expected we have in total ten functions.

The scalar perturbations are defined by scalar functions $\phi, \psi, B, E$ and perturbed metric around $F R W$ background is

$$
d s^{2}=a(\tau)^{2}\left[-(1-2 \phi) d \tau^{2}+\partial_{i} B \mathrm{~d} \tau d x^{i}+\left((1+2 \psi) \delta_{i j}+2 \partial_{i} \partial_{j} E\right) d x^{i} d x^{j}\right]
$$

The vector perturbations are defined by vectors $S_{i}$ and $F_{i}$, i.e.

$$
d s^{2}=a(\tau)^{2}\left[-d \tau^{2}+S_{i} B \mathrm{~d} \tau d x^{i}+\left(\delta_{i j}+\partial_{i} F_{j}+\partial_{j} F_{i}\right) d x^{i} d x^{j}\right] .
$$

Tensor perturbations are defined by $\varphi_{i j}$ and describe gravitational waves, which have no analog in Newton gravity theory.

$$
d s^{2}=a(\tau)^{2}\left[-d \tau^{2}+\left(\delta_{i j}+\varphi_{i j}\right) d x^{i} d x^{j}\right] .
$$

Each of the types of perturbations can be studied separately. In this form of perturbations we have

$$
\begin{aligned}
h_{\mu \nu} & =a(\tau)^{2}\left(\begin{array}{cc}
2 \phi & \partial_{i} B+S^{i} \\
\partial_{i} B+S^{i} 2 \psi \mathrm{Id}+2 \operatorname{Hess} E+\partial_{j} F_{i}+\partial_{i} F_{j}+\varphi_{i j}
\end{array}\right), \\
h & =-2 \phi+6 \psi+2 \triangle E \\
\delta R & =\frac{2}{a^{2}}\left(6 \frac{a^{\prime \prime}}{a} \phi+\triangle(\phi-2 \psi)+3 \frac{a^{\prime}}{a} \triangle\left(B+E^{\prime}\right)\right. \\
& \left.+3 \frac{a^{\prime}}{a}\left(\phi^{\prime}+3 \psi^{\prime}\right)+\triangle\left(B^{\prime}+E^{\prime \prime}\right)+3 \psi^{\prime \prime}\right) .
\end{aligned}
$$

Moreover, let $A^{\mu}=\nabla_{\nu} h^{\mu \nu}$, then 


$$
\begin{aligned}
& A^{0}=6 \frac{a^{\prime}}{a^{3}}(\phi+\psi)+\frac{1}{a^{2}} \triangle B+2 \frac{a^{\prime}}{a^{3}} \triangle E+\frac{2}{a^{2}} \phi^{\prime}, \\
& A^{i}=4 \frac{a^{\prime}}{a^{3}}\left(\partial_{i} B+S^{i}\right)+\frac{2}{a^{2}}\left(\partial_{i} \psi+\triangle \partial_{i} E\right)+\frac{1}{a^{2}}\left(\partial_{i} B^{\prime}+S^{\prime i}+\triangle F^{i}\right) .
\end{aligned}
$$

Out of 4 scalar modes only 2 are gauge invariant. The convenient gauge invariant variables (Bardeen potentials) are introduced as

$$
\Phi=\phi-\frac{1}{a}\left(a\left(B-E^{\prime}\right)\right)^{\prime}, \quad \Psi=\psi+\frac{a^{\prime}}{a}\left(B-E^{\prime}\right) .
$$

The prime denotes the differentiation with respect to the conformal time $\tau$ and the dot as before w.r.t. the cosmic time $t$.

The $(1+3)$ structure suggests to represent the perturbation quantities (which can depend on all 4 coordinates) as

$$
f(\tau, \mathbf{x})=f(\tau, k) Y(k, \mathbf{x}),
$$

where $\mathbf{x}=(x, y, z)$ and $k=|\mathbf{k}|$ comes from the definition of the $Y$-functions as spatial Fourier modes

$$
\delta^{i j} \partial_{i} \partial_{j} Y=-k^{2} Y .
$$

Then

$$
Y=Y_{0} e^{ \pm i \mathbf{k x}} .
$$

The relevant expressions for the d'Alembert operator are

$$
\square=-\frac{1}{a^{2}} \partial_{\tau}^{2}-2 \frac{a^{\prime}}{a^{3}} \partial_{\tau}+\frac{\delta^{i j} \partial_{i} \partial_{j}}{a^{2}}=-\partial_{t}^{2}-3 H \partial_{t}-\frac{k^{2}}{a^{2}},
$$

where $k=|\mathbf{k}|$.

All the expression in this subsection are valid for a generic scale factor $a$ in flat space.

\section{Concluding Remarks}

In this paper we have considered a class of nonlocal gravity models without matter given by the action in the form

$$
S=\frac{1}{16 \pi G} \int_{M}(R-2 \Lambda+P(R) \mathcal{F}(\square) Q(R)) \sqrt{-g} \mathrm{~d}^{4} x .
$$

We have derived the equations of motion for this action. We also have presented the second variation of action (130) and basics of metric perturbations.

In many research papers there are equations of motion which are special cases of our equations. In the case $P(R)=Q(R)=R$ one obtains 


$$
S=\frac{1}{16 \pi G} \int_{M}(R-2 \Lambda+R \mathcal{F}(\square) R) \sqrt{-g} \mathrm{~d}^{4} x .
$$

This nonlocal model is further elaborated in the series of papers [3, 4, 5, 6, 7, 24, 10, 11, 12, 16, 17.

The action (130) for $P(R)=R^{-1}$ and $Q(R)=R$ was introduced in 12 as a new approach to nonlocal gravity. This model one can also find in 13 .

The case $P(R)=R^{p}$ and $Q(R)=R^{q}$ we analyzed in [16, 17.

For the case $P(R)=\left(R+R_{0}\right)^{m}$ and $Q(R)=\left(R+R_{0}\right)^{m}$ see [14, 15].

Studies of this model with $R=$ const can be found in [18, 19.

It is worth noting that cosmology with nonlocality in the matter sector was also investigated, see e.g. [1, 22].

For some very recent achievements in higher derivative modified gravities one can see [23, 25, 8, 26].

Note that there is the following formula $\square^{-1}=\int_{0}^{\infty} e^{-\alpha \square} d \alpha$, which could be used in investigation of models containing $\square^{-n}, n \in \mathbb{N}$, where

$$
\square^{-n}=\frac{1}{(n-1) !} \int_{0}^{\infty} \alpha^{n-1} e^{-\alpha \square} d \alpha .
$$

Namely, formalism presented in the previous sections can easily incorporate this case taking $\mathcal{F}(\square)=e^{-\alpha \square}$ and at the end performing integration over $\alpha$.

Acknowledgements Work on this paper was partially supported by the Ministry of Education, Science and Technological Development of Republic of Serbia, grant No 174012. B.D. thanks Prof. Vladimir Dobrev for invitation to participate and give a talk on nonlocal gravity, as well as for hospitality, at the X International Symposium "Quantum Theory and Symmetries", and XII International Workshop "Lie Theory and its Applications in Physics", 19-25 June 2017, Varna, Bulgaria. B.D. also thanks a support of the ICTP - SEENET-MTP project NT-03 Cosmology-Classical and Quantum Challenges during preparation of this article.

\section{References}

1. Aref'eva, I.Ya., Joukovskaya, L.V., Vernov, S.Yu.: Bouncing and accelerating solutions in nonlocal stringy models. JHEP 0707, 087 (2007) hep-th/0701184

2. E. Belgacem, Y. Dirian, S. Foffa and M. Maggiore: Nonlocal gravity. Conceptual aspects and cosmological predictions. Journal of Cosmology and Astroparticle Physics, Volume 2018, (2018) arXiv:1712.07066 [hep-th]].

3. Biswas, T., Mazumdar, A., Siegel, W: Bouncing universes in string-inspired gravity. J. Cosmology Astropart. Phys. 0603, 009 (2006) arXiv:hep-th/0508194

4. Biswas, T., Koivisto, T., Mazumdar, A.: Towards a resolution of the cosmological singularity in non-local higher derivative theories of gravity. J. Cosmology Astropart. Phys. 1011, 008 (2010) arXiv:1005.0590 2 [hep-th]].

5. Biswas, T., Gerwick, E., Koivisto, T., Mazumdar, A.: Towards singularity and ghost free theories of gravity. Phys. Rev. Lett. 108, 031101 (2012) arXiv:1110.5249v2 [gr-qc]] 
6. Biswas, T., Conroy, A., Koshelev, A.S., Mazumdar, A.: Generalized gost-free quadratic curvature gravity. arXiv:1308.2319 [hep-th]]

7. T. Biswas, A. S. Koshelev, A. Mazumdar, S. Yu. Vernov, Stable bounce and inflation in non-local higher derivative cosmology, JCAP 08 (2012) 024, arXiv:1206.6374v2 [astro-ph.CO]].

8. L. Buoninfante, A. S. Koshelev, G. Lambiase and A. Mazumdar: Classical properties of non-local, ghost- and singularity-free gravity. arXiv:1802.00399] [gr-qc]].

9. T. Clifton, P. G. Ferreira, A. Padilla, C. Skordis: Modified gravity and cosmology. Physics Reports 513 (2012) 1-189; arXiv:1106.2476v2 [astro-ph.CO]].

10. I. Dimitrijevic, B. Dragovich, J. Grujic, Z. Rakic: On modified gravity. Springer Proceedings in Mathematics and Statistics 36, 251-259 (2013) arXiv:1202.2352 [hep-th]]

11. Dimitrijevic, I., Dragovich, B., Grujic J., Rakic, Z.: New cosmological solutions in nonlocal modified gravity. Rom. Journ. Phys. 58 (5-6), 550-559 (2013) arXiv:1302.2794 [gr-qc]]

12. I. Dimitrijevic, B. Dragovich, J. Grujic and Z. Rakic: A new model of nonlocal modified gravity. Publications de l'Institut Mathematique 94 (108) (2013), 187196.

13. I. Dimitrijevic, B. Dragovich, J. Grujic and Z. Rakic: Some power-law cosmological solutions in nonlocal modified gravity. in: Lie Theory and Its Applications in Physics, Springer Proceedings in Mathematics and Statistics, 111 2014, pp. 241-250.

14. Dimitrijevic, I., Dragovich, B., Grujic J., Koshelev A. S., Rakic, Z.: Cosmology of modified gravity with a non-local $f(R)$. arXiv:1509.04254 [hep-th]

15. I. Dimitrijevic, B. Dragovich, J. Stankovic, A. S. Koshelev and Z. Rakic: On Nonlocal Modified Gravity and Its Cosmological Solutions. Springer Proceedings in Mathematics \& Statistics 191 (2016) 35-51. arXiv:1701.02090 [hep-th].

16. I. Dimitrijevic, B. Dragovich, J. Grujic and Z. Rakic: Some Cosmological Solutions of a Nonlocal Modified Gravity. Filomat 29 (3), (2015) 619-628, arXiv:1508.05583 [hep-th].

17. I. Dimitrijevic: Cosmological solutions in modified gravity with monomial nonlocality. Applied Mathematics and Computation, 285 (3), (2016) 195-203.

18. I. Dimitrijevic, B. Dragovich, J. Grujic and Z. Rakic: Constant curvature cosmological solutions in nonlocal gravity. AIP Conference Proceedings 1634, (2014) $18-23$.

19. I. Dimitrijevic, B. Dragovich, Z. Rakic and J.Stankovic: On Nonlocal Gravity with Constant Scalar Curvature. Publications de l'Institut Mathematique, Nouvelle série, 103 (117) (2018), 53-59.

20. B. Dragovich: On Nonlocal modified gravity and cosmology. Lie Theory and Its Applications in Physics, Springer Proceedings in Mathematics and Statistics 111, 251-262, 2014.

21. Dragovich, B., Khrennikov, A. Yu., Kozyrev, S. V., Volovich, I. V., Zelenov, E. I.: $p$-Adic mathematical physics: the first 30 years. p-Adic Numbers Ultrametric Anal. Appl. 9 (2), 87-121 (2017). [arXiv:1705.04758 [math-ph]]

22. E. Elizalde, E. O. Pozdeeva, S. Yu. Vernov: Stability of de Sitter Solutions in Non-local Cosmological Models. PoS, QFTHEP2011:038, 2013, arXiv:1202.0178.

23. A. S. Koshelev, K. S. Kumar, A. A. Starobinsky: $R^{2}$ inflation to probe nonperturbative quantum gravity. Journal of High Energy Physics 1803 (2018) 071. arXiv:1711.08864 [hep-th]]

24. A. S. Koshelev, S. Yu. Vernov: On bouncing solutions in non-local gravity. Phys. Part. Nuclei 43, 666-668 (2012) arXiv:1202.1289v1 [hep-th]].

25. A. S. Koshelev, L. Modesto, L. Rachwal and A. A. Starobinsky: Occurrence of exact $R^{2}$ inflation in non-local UV-complete gravity. Journal of High Energy Physics, 2016(11), 1-41. arXiv:1604.03127 [hep-th]] 
26. A. S. Koshelev, J. Marto, A. Mazumdar: Towards non-singular metric solution in infinite derivative gravity. arXiv:1803.00309 [gr-qc]].

27. Modesto, L.: Super-renormalizable quantum gravity. Phys. Rev. D 86, 044005 (2012). arXiv:1107.2403 [hep-th]]

28. Modesto, L., Rachwal, L.: Super-renormalizable and finite gravitational theories. Nucl. Phys. B 889, 228 (2014). arXiv:1407.8036 [hep-th]]

29. V. Mukhanov, Physical Foundations of Cosmology, (Cambridge, 2005)

30. S. Nojiri, S. D. Odintsov: Unified cosmic history in modified gravity: from $F(R)$ theory to Lorentz non-invariant models. Physics Reports 505 (2011) 59-144; arXiv:1011.0544v4 [gr-qc]].

31. S. Nojiri, S. D. Odintsov, V. K. Oikonomou: Modified Gravity Theories on a Nutshell: Inflation, Bounce and Late-time Evolution. Physics Reports 692 (2017) 1-104. [arXiv:1705.11098 [gr-qc]] .

32. Novello, M., Bergliaffa, S.E.P.: Bouncing cosmologies. Phys. Rep. 463, 127-213 (2008). arXiv:0802.1634 [astro-ph]]

33. T. P. Sotiriou, V. Faraoni: $f(R)$ theories of gravity. Rev. Mod. Phys. 82 (2010) 451-497. arXiv:0805.1726 4 [gr-qc]]

34. Stelle, K.S.: Renormalization of higher derivative quantum gravity. Phys. Rev. D 16, 953 (1977).

35. R. M. Wald, General Relativity (University of Chicago Press, 1984).

36. R. P. Woodard: Nonlocal models of cosmic acceleration. arXiv:1401.0254 [astroph.CO]] (2014). 